\title{
Contact anisotropy and coordination number for a granular assembly: A comparison of distinct-element-method simulations and theory
}

\author{
Luigi La Ragione ${ }^{1}$ and Vanessa Magnanimo ${ }^{2}$ \\ ${ }^{1}$ Dipartimento di Ingegneria Civile e Ambientale, Politecnico di Bari, Via Orabona 4, 70125 Bari, Italy \\ ${ }^{2}$ Multi Scale Mechanics (MSM), CTW, UTwente, P.O. Box 217, 7500AE Enschede, The Netherlands
}

(Received 14 June 2011; published 19 March 2012)

\begin{abstract}
We study an ideal granular aggregate consisting of elastic spherical particles, isotropic in stress and anisotropic in the contact network. Because of the contact anisotropy, a confining pressure applied at zero deviatoric stress, produces shear strain as well as volume strain. Our goal is to predict the coordination number $k$, the average number of contacts per particle, and the magnitude of the contact anisotropy $\varepsilon$, from knowledge of the elastic moduli of the aggregate. We do this through a theoretical model based upon the well known effective medium theory. However, rather than focusing on the moduli, we consider their ratios over the moduli of an equivalent isotropic state. We observe good agreement between numerical simulation and theory.
\end{abstract}

DOI: 10.1103/PhysRevE.85.031304

PACS number(s): 81.05.Rm, 83.80.Fg, 81.05.Xj, 46.40.-f

\section{INTRODUCTION}

Granular materials show interesting behavior and special properties, different from classical fluids or solids [1,2]. Nonlinearity, dilatancy, loading history dependence, anisotropy [3-5], among many others, are typical features of the behavior of a granular aggregate. In this paper, we devote our attention to anisotropy because of its central role in a proper comparison between theoretical models and physical experiments. For example, when a laboratory test on sand is carried out, the stress-strain response is, in general, far from isotropic. Anisotropy is, typically, created during the preparation of the sample [6-20]. That is, preferential directions appear due to the depositional process used to prepare the sample (inherent anisotropy) or induced by the strain (induced anisotropy). In particular, inherent anisotropy is associated with the orientation of the network of contacts [7], while induced anisotropy may be characterized by two distinct effects: contact anisotropy and anisotropy in the stress distribution (e.g., Refs. [21,22]).

We restrict our attention to situations in which geometric anisotropy is dominant, while stress anisotropy is negligible. However, while there is abundant evidence of such anisotropy in physical experiments (e.g., Refs. [23-28]), a paucity of data is available from numerical simulations (e.g., Ref. [29]). This is because most of the numerical simulations involve periodic boundary conditions that eliminate preferential contact directions. In addition to a theoretical model, we consider numerical simulations in which anisotropy is present.

We attempt to model a granular material characterized by a contact anisotropy. We do this in the context of micromechanics. While there is abundant evidence of the impact of such anisotropy on the constitutive behavior, it remains a challenging task to take the anisotropic effects into account in an analytical model. This is an essential issue for a proper analysis of the aggregate. For example, predictions of the elastic moduli of an isotropic aggregate have been made in the context of effective medium theory (EMT) where particles move according to the average deformation [30,31] or within a more sophisticated theory where fluctuations are included in the description of the kinematics of contacting particles [32,33]. In both cases, given the confining pressure and the porosity, the response depends only on the coordination number (the average number of contacts per particle). However, when we deal with an anisotropic aggregate, we expect that both contact anisotropy and coordination number characterize the microstructure of the sample and its response.

We present an analysis based upon EMT in which both the coordination number $k$ and the magnitude of the contact anisotropy $\varepsilon$ are predicted from the knowledge of the effective moduli of an anisotropic aggregate. EMT has been applied already to derive the elastic moduli of a transversely isotropic aggregate (e.g., Refs. [12,34-36]). In Ref. [35], the variation in the elastic constants is given for different values of $\varepsilon$, but a comparison with numerical simulations or physical experiments is not made. On the other hand, in Ref. [36], the theoretical model is a function of three parameters associated with the orientation of the contact forces, particle rotation, deletion, and anisotropy. A comparison with physical experiments is given and shows good agreement only for some particular values of these parameters. In Ref. [20], EMT also is adopted to compare theory and physical experiments on glass beads in an oedometric test. The anisotropy is induced by the uniaxial compression, but an inherent anisotropy also is present because of the preparation process and the boundary conditions of the sample. Stress and contact anisotropy are not distinguished in the model. With this hypothesis, the agreement between theory and physical experiments is good when a high coordination number is considered.

Here, we use EMT analysis in a slightly different way. Instead of considering the effective moduli themselves, we focus on their ratios over those of an isotropic state. We show that, while the prediction of the effective moduli based upon the EMT fails, the prediction of the ratios is in better agreement with numerical simulations. In order to support this, we develop a numerical tool to generate contact anisotropy and compare the model against numerical simulations. It is a nontrivial task to create a dense anisotropic numerical state under a given pressure $p$ and zero deviatoric stress $q$. To our knowledge, numerical simulations of granular material have dealt with anisotropy only when a deviatoric stress and/or gravity are present. Here, neither deviatoric stress nor gravity has been employed, and a particular loading path has been developed to generate a sample characterized only by contact 
anisotropy. Despite the simplicity of the model, we find good agreement between the simulation and the EMT ratio. This is interesting because so simple a theory may provide insight into the microstructure parameters of real granular aggregates when acoustic measurements are available.

In this paper, we introduce a procedure to create numerical samples characterized only by geometrical anisotropy under different confining pressures with $q=0$. Because of the preparation process, the aggregates are transversely isotropic; each one has a different coordination number $k$ and a different degree of anisotropy $\varepsilon$. For each packing, the effective moduli are inferred. Next, we derive $k$ and $\varepsilon$ from the EMT ratios and compare the theoretical prediction with the numerical simulation.

\section{NUMERICAL SIMULATION}

In recent years, theoretical models and experiments have been complemented by numerical simulations. However, simulations have concentrated on stress anisotropy rather than geometric anisotropy [37] or on conditions in which both are present. This makes their individual contributions indistinguishable [38]. The geometric anisotropy is isolated from that of the stress $[27,28]$, and it can be shown that the two phenomena are independent. Our goal is to create numerical samples in which the geometric anisotropy is isolated from the stress in order to understand the effect of the fabric alone on the elastic behavior of a granular sample. We use distinct element method (DEM) simulations ( [39]) on random assemblies of identical frictional elastic spheres. Our numerical experiments consider $N=2000$ particles, each with diameter $D=0.2 \mathrm{~mm}$, randomly generated in a periodic cubic cell of approximately $3 \mathrm{~mm}$ on a side. We employ material properties typical of glass spheres: shear modulus $\mu=29 \mathrm{GPa}$ and Poisson's ratio $v=0.2$. The interaction between particles is given by a noncentral contact force $\mathbf{F}$ where the normal component follows the nonlinear Hertz law. The tangential component is bilinear: an initial elastic displacement followed by Coulomb sliding (e.g., Ref. [40]). We want to create dense packings with a given confining pressure $p$ characterized by stress isotropy and geometrical anisotropy. To achieve these complex configurations, the properties are set in different phases during the loading path.

\section{A. Contact anisotropy}

It is difficult to create geometrical anisotropy in a numerical specimen with periodic boundary conditions. Here, we consider a particular protocol to obtain very dense packings characterized by a contact anisotropy. The goal is to reproduce the consolidation process that occurs before any test when a laboratory sample is filled with a granular material and is confined under a given pressure.

After random generation in a periodic box, gravity-free particles are compressed isotropically from an initial gas to the desired solid volume fraction. In order to reach this final dense state, the friction between particles is removed. The compression is stopped when the sample has reached a solid volume fraction $\phi=0.607$. Then, the particles are allowed to relax. At the end of this relaxation process, both pressure and coordination numbers are zero. This is the reference state for the sample

We label, with $\mathbf{e}_{1}, \mathbf{e}_{2}$, and $\mathbf{e}_{3}$, the three orthogonal vectors of a coordinate system; a quasistatic uniaxial compression along $\mathbf{e}_{3}$ is applied with particle friction coefficient $\mu_{1}=0.1$ and the stresses are set to $\sigma_{11}=\sigma_{22}=\sigma_{33}=50 \mathrm{kPa}$. This stresscontrolled deformation is carried out using a servomechanism that constantly adjusts the applied strain rate according to the difference between the desired stress state $\sigma_{i j}^{*}$ and the measured stress state $\sigma_{i j}$. At each time step, the strain rates $\dot{\varepsilon}_{i j}$ are adjusted to the value,

$$
\dot{\varepsilon}_{i j}^{s}=\dot{\varepsilon}_{i j}+g\left(\sigma_{i j}-\sigma_{i j}^{*}\right),
$$

where $g$ is a gain factor that is tuned to achieve equilibrium in an optimal way. At this point, we change the particle friction coefficient from $\mu_{1}$ to $\mu_{2}$ and additional servocontrolled cycles are applied at a constant volume. $\mu_{2}$ assumes different values as reported in Table I, and, for each $\mu_{2}$, different samples, each with different micromechanical characteristics, are obtained $\left(S_{1}, S_{2}, S_{3}, S_{4}\right)$. Finally, we remove the servomechanism, fix the friction coefficient at a very high value for all packings $\left(\mu_{f}=\right.$ 10.0 ), and let the grains relax to equilibrium. The solid volume fraction reaches $\phi=0.635$, slightly lower than random close packing $\left(\phi_{\mathrm{RCP}} \simeq 0.64\right.$ for monodisperse aggregates [41]). In Table I, we report the macroscopic and micromechanical properties of the four samples obtained by this process under a confining pressure of $50 \mathrm{kPa}$.

Each of the four packings $\left(S_{1}, S_{2}, S_{3}, S_{4}\right)$ is isotropically compressed to $p=100, p=250$, and $p=500 \mathrm{kPa}$ with the deviatoric stress $q=0$. The compression is carried out with a friction coefficient $\mu_{2}$, which is different for each packing, as previously underlined. In so doing, we obtain 16 packings under different confining pressures, each with zero deviatoric stress and an anisotropic distribution of contacts, characterized by a preferential direction $\mathbf{e}_{3}$.

\section{B. Microstructure and elastic moduli}

We use a DEM simulation to scrutinize the contact network of our numerical samples. We focus on the coordination

TABLE I. Macroscopic and micromechanical properties of the numerical samples at a confining pressure of $50 \mathrm{kPa}$.

\begin{tabular}{lcccccccccc}
\hline \hline Samples & $\mu_{1}$ & $\mu_{2}$ & $\sigma_{11}$ & $\sigma_{22}$ & $\sigma_{33}$ & $k$ & $L_{11}$ & $L_{22}$ & $L_{33}$ & $\phi$ \\
\hline$S_{1}$ & 0.1 & 0.05 & 49.99 & 49.98 & 49.99 & 5.58 & 0.330 & 0.329 & 0.341 \\
$S_{2}$ & 0.1 & 0.10 & 50.03 & 49.99 & 49.94 & 5.27 & 0.328 & 0.326 & 0.346 & 0.636 \\
$S_{3}$ & 0.1 & 0.30 & 50.00 & 50.01 & 50.00 & 4.87 & 0.321 & 0.323 & 0.356 & 0.636 \\
$S_{4}$ & 0.1 & 0.15 & 50.02 & 50.03 & 50.05 & 4.95 & 0.324 & 0.323 & 0.353 & 0.636 \\
\hline \hline
\end{tabular}




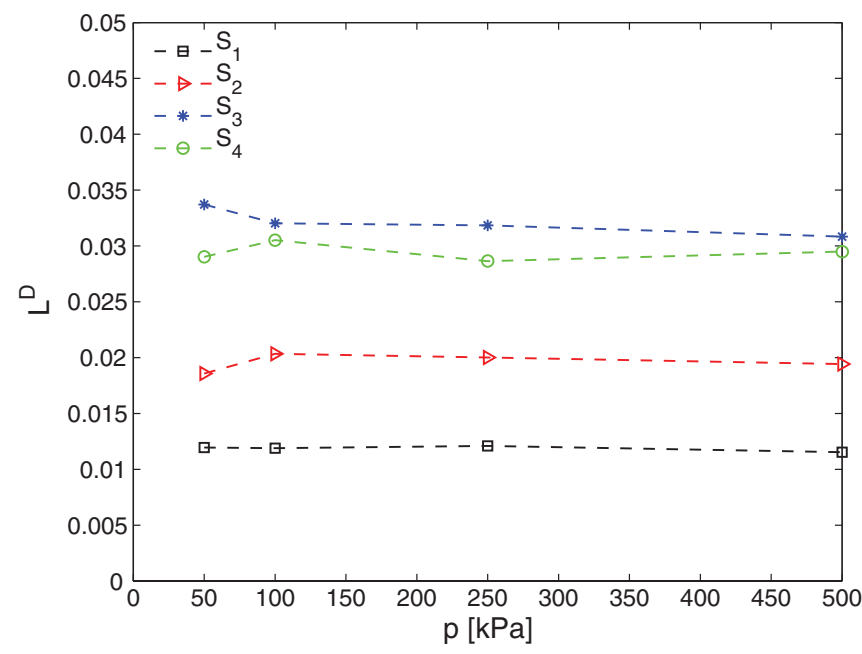

FIG. 1. (Color online) Evolution of the deviatoric fabric with pressure for the anisotropic states.

number and the fabric tensor $[7,8]$ defined as

$$
L_{i j}=\frac{1}{N^{c}} \sum_{p=1}^{N^{c}} \hat{d}_{i}^{p} \hat{d}_{j}^{p},
$$

where $N^{c}$ is the total number of contacts in the aggregate and $\hat{d}_{i}^{p}$ are direction cosines of the $p$ th contact. The second order tensor $\mathbf{L}$ is symmetric, and its trace is equal to 1 . While $k$ is a measure of the contacts density in the aggregate, the fabric tensor $\mathbf{L}$ provides information on the spatial distribution of the contact vectors through its eigenvalues. We calculate the eigenvalues $L_{i i}$ in the various states to show the degree of anisotropy in the aggregates, and we verify that fabric and strain are almost collinear in the applied range of deformation (the off-diagonal components of the fabric are orders of magnitude less than the diagonal terms).

All the states are characterized by stress isotropy and an anisotropic distribution of contacts. Due to the initial uniaxial compression, the states are transversally isotropic with $L_{11} \simeq L_{22}$. In Fig. 1, we plot a measure of the deviatoric fabric,

$$
L^{D}=L_{33}-\frac{L_{11}+L_{22}}{2}
$$

versus the pressure. Packings created with higher $\mu_{2}$ (see Table I) have a lower coordination number $k$ and are more anisotropic, i.e., show a higher deviatoric fabric. When the pressure increases for a given initial state, the geometric anisotropy is almost constant.

We next study the influence of the geometric anisotropy on the elastic properties of the granular assembly. For each state, we apply an incremental strain to the sample, we allow it to relax toward an equilibrated state, and we measure the effective moduli of the aggregate $[40,42]$. The friction coefficient is set at a very high value to prevent sliding between the grains. For each elastic response, we perform the calculation under different strain amplitudes to identify the linear regime. We note that solid volume fraction and coordination number do not vary during the applied increment of deformation and the subsequent relaxation toward the equilibrium. In Fig. 2, we plot the two shear moduli. Both $\bar{G}_{12}$ and $\bar{G}_{13}$ increase

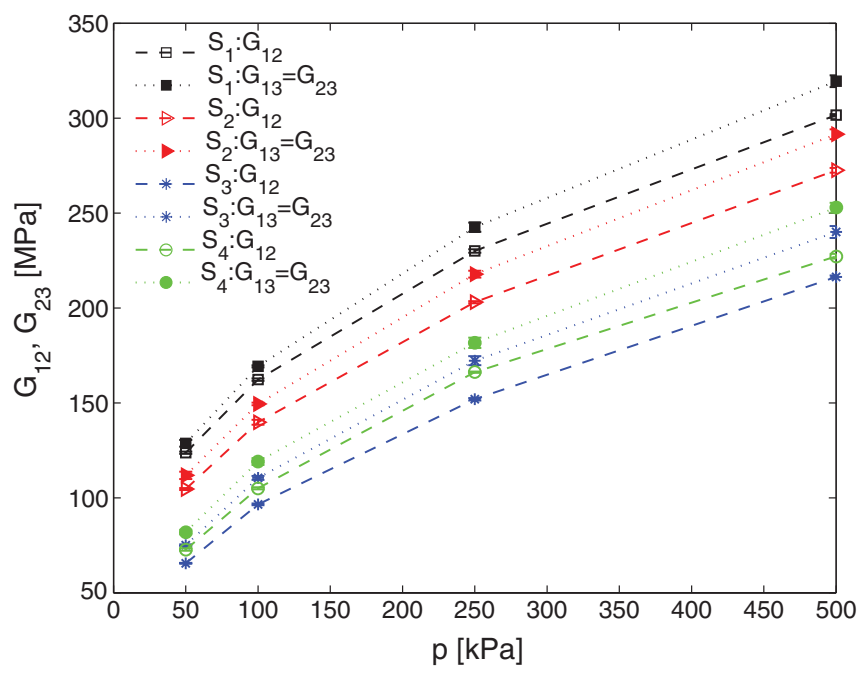

FIG. 2. (Color online) Evolution of the axial and transversal shear moduli with pressure for the anisotropic states.

with pressure and coordination number. The shear stiffness is different in the transverse and axial directions and, because the stress is isotropic, we deduce that the fabric affects the load-carrying behavior: The difference $\bar{G}_{12}-\bar{G}_{13}$ depends on the geometric anisotropy. We also notice that $\bar{G}_{12}$ and $\bar{G}_{13}$ increase with increasing pressure and constant $L^{D}$. This is because increasing the pressure with identical deviatoric fabric induces an increase in shear strain $\gamma$. Both $L^{D}$ and $\gamma$ are sources of anisotropy.

With this information about micro- and macroproperties of the aggregate, we next focus on the theoretical model.

\section{THEORY}

The magnitude of anisotropy in a granular aggregate often is associated with macroscopic measurements, such as the difference between $\bar{G}_{13}$ and $\bar{G}_{12}$ or between the Young moduli in the axial and the radial directions [36]. We develop a theoretical model to characterize the microstructure of the sample in terms of contact anisotropy and coordination number.

We consider an ideal granular material made of a random aggregate of identical elastic glass spheres with diameter $D$. Following the main assumption of the EMT, we assume that the contact displacement $\mathbf{u}$ between particles is given in terms of the average strain $\mathbf{E}$,

$$
u_{i}=\frac{D}{2} E_{i j} \hat{d}_{j}
$$

where $\hat{\mathbf{d}}$ is the unit contact vector whose components are ( $\sin \theta \cos \psi, \sin \theta \sin \psi, \cos \theta$ ) and $\theta$ is the polar angle from the axis of symmetry. In the case of homogeneous deformation, we employ the expression for the stress in terms of the contact force and the orientational distribution function [43],

$$
\sigma_{i j}=-\frac{n D}{2} \int_{\Omega} A(\hat{\mathbf{d}}) F_{i} \hat{d}_{j} d \Omega,
$$

where $n$ is the number of particles per unit volume, $A(\hat{\mathbf{d}})$ is the distribution function for contact orientations, defined so that 
its integral over the entire solid angle $\Omega$ is given by

$$
\int_{\Omega} A(\hat{\mathbf{d}}) d \Omega=k,
$$

and $\mathbf{F}$ is the contact force as previously defined. Following Ref. [44], we introduce the geometric anisotropy through the unit vector $\mathbf{h}$ in the direction of the axis of anisotropy and the strength $\varepsilon$ of the anisotropy. The approximation of the distribution function is $[45,46]$

$$
A(\hat{\mathbf{d}})=\frac{k}{4 \pi}\left[(1-\varepsilon)+3 \varepsilon\left(h_{i} \hat{d}_{i}\right)^{2}\right]
$$

\section{A. Isotropic compression}

As in the DEM simulations, we consider a stress-controlled isotropic test for the anisotropic aggregate in which we control the confining pressure $p=-\sigma_{k k} / 3$ with the condition that the deviatoric stress $q=-\left(\sigma_{33}-\sigma_{11}\right)$ is maintained at zero. Because of the anisotropy, when an isotropic pressure is applied, with zero deviatoric stress, both shear strain $\gamma=-\left(E_{11}-E_{33}\right) / 2$ and volume strain $\Delta=-\left(2 E_{11}+E_{33}\right)$ (positive in compression) occur. Therefore, $\gamma$ is a measure of the anisotropy. We also recall that the confining pressure is applied to a very dense state in which the solid volume fraction is about 0.64 . Under these circumstances, particles are in contact and, with increasing pressure, we assume an elastic response and neglect particle sliding and deletion.

\section{B. Incremental response}

For any of these anisotropic states, we consider the elastic response to increments in strain. That is, we exclude sliding and deletion between particles, and we determine the effective moduli. This assumption is not strictly true as numerical simulations show the presence of both deletion and sliding [47]. Both mechanisms, however, involve a small percentage of weakly loaded contacts, and consequently, their contribution can be neglected.

The incremental contact force is written in terms of the contact stiffness $K_{N}$ and $K_{T}$,

$$
\dot{F}_{i}=\left[K_{N} \hat{d}_{j} \hat{d}_{i}+K_{T}\left(\delta_{i j}-\hat{d}_{j} \hat{d}_{i}\right)\right] \dot{u}_{j}
$$

with

$$
K_{N}=\frac{2^{3 / 2} \mu D^{1 / 2}}{1-v} \delta^{1 / 2},
$$

and

$$
K_{T}=\frac{2^{5 / 2} \mu D^{1 / 2}}{2-v} \delta^{1 / 2},
$$

in which $\delta=-D\left(E_{i j} \hat{d}_{j} \hat{d}_{i}\right) / 2$ is the normal component of the contact displacement. During the isotropic compression, $\gamma$ is typically small compared to $\Delta$ [48]. Therefore, if we write the strain as the sum of its isotropic and deviatoric parts,

$$
E_{i j}=-\frac{\Delta}{3} \delta_{i j}+\hat{E}_{i j}
$$

with $\hat{E}_{k k}=0$, and

$$
\hat{E}_{i j}=-\frac{2}{3} \gamma \delta_{i j}+2 \gamma h_{i} h_{j}
$$

we can derive, by retaining only terms that are linear in the ratio of the deviatoric to the isotropic strain, the expression for the normal component of the contact displacement,

$$
\delta^{1 / 2} \simeq\left(\frac{D \Delta}{6}\right)^{1 / 2}\left(1-\frac{3}{2} \frac{\hat{E}_{i j} \hat{d}_{j} \hat{d}_{i}}{\Delta}\right) .
$$

In this way, the reference state enters in the stiffness through Eq. (9) and is a function of both $\Delta$ and $\gamma$. This is important because micromechanical models applied to anisotropic aggregates neglect the contribution from $\gamma$. The incremental stress-strain relation is simply

$$
\dot{\sigma}_{i j}=\frac{n D}{2} \int_{\Omega} A(\hat{\mathbf{d}}) K_{T} \dot{u}_{i} \hat{d}_{j} d \Omega,
$$

where a simplification has been adopted. In fact, because we refer to an aggregate of glass beads $(v=0.2)$, we neglect terms proportional to the difference in the stiffness $K_{N}-$ $K_{T} \sim v /[(1-v)(2-v)]$ with respect to those proportional to $K_{T} \sim 2 /(2-v)$. This assumption has been tested, and we avoid reporting the results for the sake of simplicity. After we carry out the integral (the results are given in Appendix A), Eq. (11) can be written in a compact form as

$$
\dot{\sigma}_{i j}=B_{i j k l} \dot{E}_{k l}
$$

with

$$
\begin{aligned}
B_{i j k l}= & \psi_{1} h_{i} h_{j} h_{k} h_{l}+\psi_{2} \delta_{i j} \delta_{k l}+\psi_{3}\left(\delta_{i k} \delta_{j l}+\delta_{i l} \delta_{j k}\right) \\
& +\psi_{4}\left(\delta_{k l} h_{i} h_{j}+h_{l} h_{k} \delta_{i j}\right) \\
& +\psi_{5}\left(\delta_{i k} h_{j} h_{l}+\delta_{j l} h_{k} h_{i}+\delta_{i l} h_{j} h_{k}+\delta_{j k} h_{i} h_{l}\right),
\end{aligned}
$$

where the $\psi$ are the five independent elastic moduli for a transversely isotropic material,

$$
\begin{gathered}
\psi_{1}=\frac{n D^{3} \mu k}{4-2 v}\left(\frac{\Delta}{3}\right)^{1 / 2}\left(\frac{4 \varepsilon}{5}-a \frac{28+16 \varepsilon}{35} \frac{\gamma}{\Delta}\right) \\
\psi_{3}=\frac{n D^{3} \mu k}{4-2 v}\left(\frac{\Delta}{3}\right)^{1 / 2}\left(\frac{1}{3}-\frac{2}{15} \varepsilon+\frac{14-20 \varepsilon}{105} \frac{\gamma}{\Delta}\right) \\
\psi_{5}=\frac{n D^{3}}{2} \frac{\mu}{2-v}\left(\frac{\Delta}{3}\right)^{1 / 2} k\left(\frac{1}{3}-\frac{2}{15} \varepsilon+\frac{14-20 \varepsilon}{105} \frac{\gamma}{\Delta}\right) a
\end{gathered}
$$

and

$$
\psi_{2}=\psi_{4}=0
$$

with

$$
a=\frac{\frac{4 \varepsilon}{5}-\left(\frac{16}{35} \varepsilon+\frac{4}{5}\right) \frac{\gamma}{\Delta}}{\frac{4}{3}+\frac{4}{15} \varepsilon-\left(\frac{128}{105} \varepsilon+\frac{4}{15}\right) \frac{\gamma}{\Delta}} .
$$

These expressions clearly show how the two anisotropic terms $\varepsilon$ and $\gamma$, along with $k$ and $\Delta$, enter in the macroscopic response of the aggregate. A relation among the $\psi$, the two Young's moduli, the shear moduli, and the Poisson ratio can easily be obtained. However, our goal is not to compare the elastic moduli derived by the theoretical model with those inferred from numerical simulations. It is known, in fact, that the EMT overpredicts the elastic response of the aggregate with respect to numerical simulation $[40,47,49]$. Here, we attempt to predict $k$ and $\varepsilon$ of a transversely isotropic aggregate by 
TABLE II. Deviatoric over volumetric strain of the numerical samples $S_{1}-S_{4}$ for different values of the confining pressure in the reference state.

\begin{tabular}{lcccr}
\hline \hline Sample & $50 \mathrm{kPa}$ & $100 \mathrm{kPa}$ & $250 \mathrm{kPa}$ & $500 \mathrm{kPa}$ \\
\hline$S_{1}$ & -0.0623 & -0.0603 & -0.0577 & -0.0545 \\
$S_{2}$ & -0.0730 & -0.0711 & -0.0678 & -0.0643 \\
$S_{3}$ & -0.0759 & -0.0745 & -0.0719 & -0.0691 \\
$S_{4}$ & -0.0763 & -0.0750 & -0.0724 & -0.0695 \\
\hline \hline
\end{tabular}

assuming that the ratios of the elastic moduli, rather than the moduli themselves, are captured by so crude a theory. We consider the two shear moduli $\bar{G}_{13}, \bar{G}_{12}$ and the bulk modulus $\bar{\Theta}^{\text {ani }}$ of the anisotropic aggregate and the elastic moduli of an equivalent isotropic state (with $\varepsilon=0, \gamma=0) \bar{G}$ and $\bar{\Theta}$ (see Appendix A). The equivalence between an anisotropic and an isotropic state is based upon the assumption that both states have the same pressure and volume but may have different coordination numbers, respectively, $k$ and $\hat{k}$. The result is

$$
\begin{gathered}
\frac{\bar{\Theta}^{\mathrm{ani}}}{\bar{\Theta}}=\frac{k}{\hat{k}}\left[(3+2 a)\left(\frac{1}{3}-\frac{2}{15} \varepsilon+\frac{14-20 \varepsilon}{105} \frac{\gamma}{\Delta}\right)\right. \\
\left.+\left(\frac{2 \varepsilon}{5}-a \frac{14+8 \varepsilon}{35} \frac{\gamma}{\Delta}\right)\right], \\
\frac{\bar{G}_{12}}{\bar{G}}=\frac{k}{\hat{k}}\left(1-\frac{2}{5} \varepsilon+\frac{14-20 \varepsilon}{35} \frac{\gamma}{\Delta}\right),
\end{gathered}
$$

and

$$
\frac{\bar{G}_{13}}{\bar{G}}=\frac{k}{\hat{k}}\left(1-\frac{2}{5} \varepsilon+\frac{14-20 \varepsilon}{35} \frac{\gamma}{\Delta}\right)(1+a),
$$

where the unknowns are $\hat{k}, k$, and $\varepsilon$, given the elastic moduli and the ratio $\gamma / \Delta . \gamma$ and $\Delta$ are measured in the numerical simulations with respect to the reference state defined in Sec. II A (see Table II). In Appendix B, we provide more details of the normalization adopted here.

\section{COMPARISON OF THEORY AND SIMULATION}

We first derive the strength of the anisotropy of numerical samples. We employ a continuous version of Eq. (2), and from Eq. (6), normalized by $k$, we obtain

$$
L_{i j}=\int_{\Omega} \frac{1}{4 \pi}\left[(1-\varepsilon)+3 \varepsilon\left(h_{k} d_{k}\right)^{2}\right] \hat{d}_{i} \hat{d}_{j} d \Omega,
$$

or, after we carry out the integration,

$$
L_{i j}=\frac{1}{2}\left[\left(\frac{2}{3}-\frac{4}{15} \varepsilon\right) \delta_{i j}+\frac{4}{5} \varepsilon h_{i} h_{j}\right] .
$$

Knowledge of the fabric components from the numerical simulations allows us to determine $\varepsilon$ through Eq. (20). The results are reported in Table III under the column $\epsilon_{\text {sim }}$.

In Eqs. (16)-(18), we know $\bar{G}_{13}, \bar{G}_{12}, \bar{\Theta}^{\text {ani }}$ from numerical simulations, and for $\bar{G}$ and $\bar{\Theta}$, we take the expressions obtained by fitting numerical data in Fig. 8 of Ref. [42] with $\hat{k}>4.8$,

$$
\bar{G}=\alpha(\hat{k}+\beta) p^{1 / 3},
$$

TABLE III. Micromechanical properties of the samples $S_{1}-S_{4}$ from simulations and theory. Here, we report the mean values of $k$ and $\epsilon$.

\begin{tabular}{lccccccc}
\hline \hline Samples & $p(\mathrm{kPa})$ & $k_{\text {sim }}$ & $k_{\text {EMT }}$ & $k_{\text {ratio }}$ & $\hat{k}$ & $\epsilon_{\text {sim }}$ & $\epsilon_{\text {ratio }}$ \\
\hline$S_{1}$ & 50 & 5.58 & 0.31 & 5.73 & 5.63 & 0.030 & 0.016 \\
& 100 & 5.65 & 0.41 & 5.78 & 5.77 & 0.030 & 0.012 \\
& 250 & 5.74 & 0.58 & 5.89 & 5.87 & 0.030 & 0.025 \\
& 500 & 5.84 & 0.75 & 6.00 & 5.94 & 0.029 & 0.039 \\
$S_{2}$ & 50 & 5.27 & 0.27 & 5.44 & 5.31 & 0.046 & 0.031 \\
& 100 & 5.37 & 0.36 & 5.55 & 5.44 & 0.051 & 0.039 \\
& 250 & 5.48 & 0.52 & 5.68 & 5.58 & 0.050 & 0.047 \\
& 500 & 5.59 & 0.69 & 5.79 & 5.73 & 0.048 & 0.044 \\
$S_{3}$ & 50 & 4.87 & 0.18 & 4.92 & 4.49 & 0.084 & 0.171 \\
& 100 & 4.98 & 0.26 & 5.11 & 4.73 & 0.080 & 0.168 \\
& 250 & 5.11 & 0.41 & 5.31 & 4.99 & 0.080 & 0.154 \\
& 500 & 5.25 & 0.56 & 5.47 & 5.22 & 0.077 & 0.120 \\
$S_{4}$ & 50 & 4.96 & 0.19 & 5.03 & 4.63 & 0.072 & 0.153 \\
& 100 & 5.05 & 0.28 & 5.21 & 4.87 & 0.076 & 0.142 \\
& 250 & 5.19 & 0.43 & 5.37 & 5.19 & 0.071 & 0.094 \\
& 500 & 5.31 & 0.59 & 5.56 & 5.31 & 0.073 & 0.112 \\
\hline \hline
\end{tabular}

and

$$
\bar{\Theta}=\rho(\hat{k}+\zeta) p^{1 / 3},
$$

with $\alpha=170, \beta=-3.66, \rho=75, \zeta=-0.5$, and $\bar{G}, \bar{\Theta}$, and $p$ expressed in megapascals. At this point, with Eqs. (21) and (22) in Eqs. (16)-(18) and the knowledge of $\gamma / \Delta$, we derive $\hat{k}, k$, and $\varepsilon$. The results are plotted in Figs. 3 and 4 and are summarized in Table III. In particular, in Fig. 3, we compare the coordination number of the numerical simulations $k_{\text {sim }}$ to that of the theory based upon the ratio $k_{\text {ratio }}$ and that of the simple EMT $k_{\mathrm{EMT}}$. In Fig. 4, we compare the $\epsilon$ obtained in numerical simulations with the results of the model. In this case, both the EMT and the EMT ratio give the same value, although the model takes the presence of $\gamma$ in the reference state into account. Figure 3 shows the

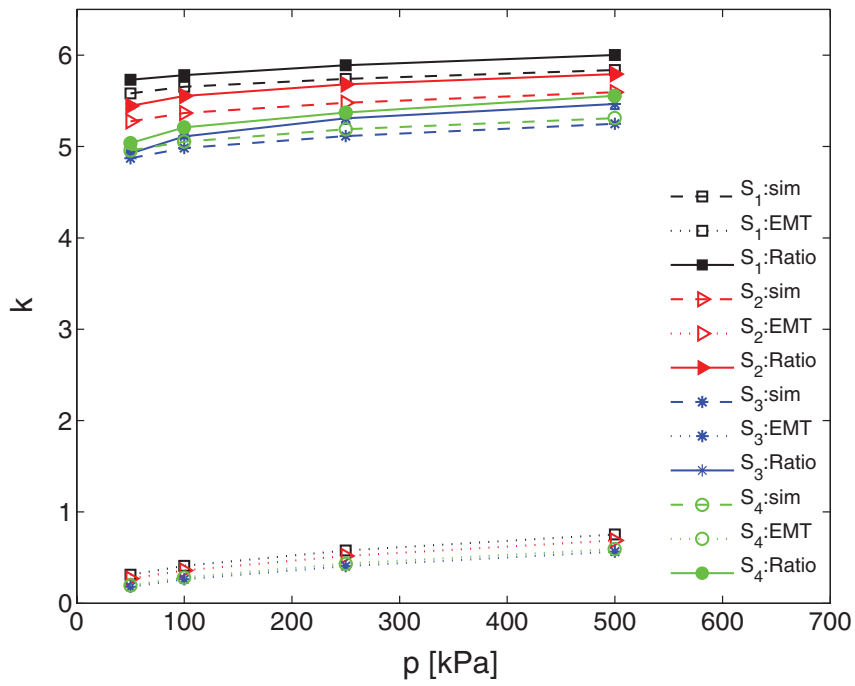

FIG. 3. (Color online) Evolution of the coordination number with increasing pressure for the anisotropic states. See Table II. 


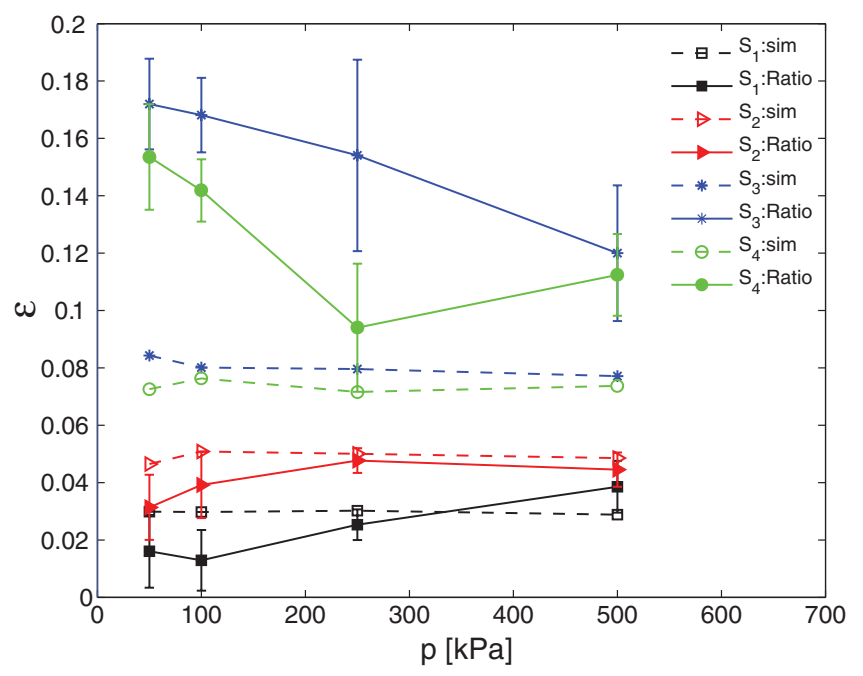

FIG. 4. (Color online) Evolution of the magnitude of anisotropy with increasing pressure for the anisotropic states. See Table II.

relevant improvement introduced by the EMT ratio, although there is still a slight difference. In Fig. 4, it appears that $\epsilon$ is sometimes overpredicted; however, for most of the samples, the comparison is reasonable. In the case of $\epsilon$, the dependence of the stiffness upon $\gamma$, see Eqs. (9) and (10), seems to be necessary to achieve good agreement between theory and simulation. In Table III, we report the coordination number $\hat{k}$ of the equivalent isotropic aggregate. It is interesting that $\hat{k}$ may differ from $k$ as anisotropy develops in the aggregate; this is an important point to take into account when comparing theory, simulation, and physical experiments. For example, in Refs. [20,47], it is assumed that an anisotropic aggregate has the same coordination number as an isotropic sample. We believe that this assumption should be tested.

\section{CONCLUSION}

Starting from the well known EMT, a tool has been developed to explore the microstructure of a granular aggregate. Instead of focusing on the moduli of a transversely isotropic aggregate, we have considered their ratios over those of an equivalent isotropic aggregate. These ratios provide the relations between the elastic moduli, the coordination number, and the strength of the contact anisotropy. At the same time, we have designed a proper numerical protocol to create granular packings characterized by only contact anisotropy. The coordination number and the strength of anisotropy of the aggregates can be measured in DEM simulations. Comparison of the EMT ratio theory with numerical simulations show that both parameters are rather well predicted. This indicates that EMT employed in so simple a model may still be used to obtain interesting information about the micromechanics of a granular material.

\section{ACKNOWLEDGMENTS}

V. Magnanimo would like to thank S. Luding and M. Liu for helpful discussions. L. La Ragione thanks MIUR (Italy) through the project COFIN 2008 "Complex materials and structural models in advanced problems of engineering."

\section{APPENDIX A}

The incremental relation for the stress is

$$
\dot{\sigma}_{i j}=\frac{n D}{2} \int_{\Omega} A(\hat{\mathbf{d}}) K_{T} \dot{u}_{i} \hat{d}_{j} d \Omega,
$$

or, with Eqs. (9) and (10), and the incremental displacement,

$$
\dot{u}_{i}=\frac{D}{2}\left(\dot{E}_{i k}+\dot{W}_{i k}-\dot{\Omega}_{i k}\right) \hat{d}_{k}
$$

we obtain

$$
\begin{aligned}
\dot{\sigma}_{i j}= & \frac{n k D^{3}}{4 \pi} \frac{\mu}{2-v}\left(\frac{\Delta}{3}\right)^{1 / 2} \int_{\Omega}\left(1-\varepsilon+3 \varepsilon \hat{d}_{q} \hat{d}_{l} h_{q} h_{l}\right) \\
& \times\left(1-\frac{3}{2} \frac{\hat{E}_{m s}}{\Delta} \hat{d}_{m} \hat{d}_{s}\right) \hat{d}_{j} \hat{d}_{k} d \Omega\left(\dot{E}_{i k}+\dot{W}_{i k}-\dot{\Omega}_{i k}\right) .
\end{aligned}
$$

It is necessary to distinguish between the incremental average rotation based upon the displacements of the particle centers $\dot{\mathbf{W}}$ and the incremental average spin about the centers $\dot{\boldsymbol{\Omega}}$ because, as anisotropies develop in the state of the material, these need not be equal. Their difference then is determined by the requirement that the stress be symmetric (e.g., Ref. [34]). We carry out the integration using the following identities:

$$
\int_{\Omega} \hat{d}_{i} \hat{d}_{l} \hat{d}_{k} \hat{d}_{j} d \Omega=\frac{4 \pi}{15} X_{i l k j},
$$

and

$$
\int_{\Omega} \hat{d}_{q} \hat{d}_{l} \hat{d}_{z} \hat{d}_{s} \hat{d}_{j} \hat{d}_{k} d \Omega=\frac{4 \pi}{105} Y_{q z s l k j},
$$

with

$$
X_{i l k j}=\delta_{i l} \delta_{k j}+\delta_{i k} \delta_{l j}+\delta_{i j} \delta_{k l},
$$

and

$$
\begin{aligned}
Y_{q z s l k j}= & \delta_{q z} X_{s l k j}+\delta_{q s} X_{z l k j}+\delta_{q l} X_{s z k j} \\
& +\delta_{q k} X_{z s l j}+\delta_{q j} X_{z s l k} .
\end{aligned}
$$

We obtain

$$
\begin{aligned}
\dot{\sigma}_{i j}= & \frac{n k D^{3}}{2} \frac{\mu}{2-v}\left(\frac{\Delta}{3}\right)^{1 / 2}\left[\frac{2}{3}(1-\varepsilon)\left(\dot{E}_{i j}+\dot{W}_{i j}-\dot{\Omega}_{i j}\right)\right. \\
& -(1-\varepsilon) \frac{\hat{E}_{z s}}{\Delta} \frac{1}{5} X_{z s j k}\left(\dot{E}_{i k}+\dot{W}_{i k}-\dot{\Omega}_{i k}\right) \\
& +\frac{2 \varepsilon}{5} X_{q l j k} h_{q} h_{l}\left(\dot{E}_{i k}+\dot{W}_{i k}-\dot{\Omega}_{i k}\right) \\
& \left.-\frac{3}{35} \varepsilon h_{q} h_{l} \frac{\hat{E}_{z s}}{\Delta} Y_{q l z s j k}\left(\dot{E}_{i k}+\dot{W}_{i k}-\dot{\Omega}_{i k}\right)\right]
\end{aligned}
$$


The symmetry of the stress requires

$$
\varepsilon_{m i j} \dot{\sigma}_{i j}=0
$$

or

$$
\begin{aligned}
0= & {\left[\varepsilon_{m i j} \frac{2}{3}(1-\varepsilon)\left(\dot{E}_{i j}+\dot{W}_{i j}-\dot{\Omega}_{i j}\right)\right.} \\
& -(1-\varepsilon) \frac{2 \hat{E}_{j k}}{\Delta} \frac{1}{5} \varepsilon_{m i j}\left(\dot{E}_{i k}+\dot{W}_{i k}-\dot{\Omega}_{i k}\right) \\
& +\frac{2 \varepsilon}{5} \varepsilon_{m i j} X_{q l j k} h_{q} h_{l}\left(\dot{E}_{i k}+\dot{W}_{i k}-\dot{\Omega}_{i k}\right) \\
& \left.-\frac{3}{35} \varepsilon h_{q} h_{l} \frac{\hat{E}_{z s}}{\Delta} Y_{q l z s j k} \varepsilon_{m i j}\left(\dot{E}_{i k}+\dot{W}_{i k}-\dot{\Omega}_{i k}\right)\right] .
\end{aligned}
$$

The solution of this is

$$
\dot{W}_{i k}-\dot{\Omega}_{i k}=a \varepsilon_{i q k} \varepsilon_{q t z} h_{s} h_{z} \dot{E}_{t s},
$$

with

$$
a=\frac{\frac{4 \varepsilon}{5}-\left(\frac{16}{35} \varepsilon+\frac{4}{5}\right) \frac{\gamma}{\Delta}}{\frac{4}{3}+\frac{4}{15} \varepsilon-\left(\frac{128}{105} \varepsilon+\frac{4}{15}\right) \frac{\gamma}{\Delta}} .
$$

The appropriate incremental symmetric stress is then,

$$
\begin{aligned}
\dot{\sigma}_{i j}= & \frac{n k D^{3}}{2} \frac{\mu}{2-v}\left(\frac{\Delta}{3}\right)^{1 / 2}\left[\frac{2}{3}(1-\varepsilon) \delta_{j t} \delta_{i s} \dot{E}_{\mathrm{st}}\right. \\
& +\frac{2}{3}(1-\varepsilon) a \varepsilon_{i q j} \varepsilon_{q t z} h_{s} h_{z} \dot{E}_{\mathrm{ts}}+\frac{2 \varepsilon}{5} X_{q l j k} h_{q} h_{l} \dot{E}_{i k} \\
& +\frac{2 \varepsilon a}{5} X_{w l j k} h_{w} h_{l} \varepsilon_{i q k} \varepsilon_{q t z} h_{s} h_{z} \dot{E}_{t s} \\
& -(1-\varepsilon) \frac{\hat{E}_{z s}}{\Delta} \frac{1}{5} X_{z s j k} \dot{E}_{i k} \\
& -(1-\varepsilon) \frac{\hat{E}_{z w}}{\Delta} \frac{a}{5} X_{z w j k} \varepsilon_{i q k} \varepsilon_{q t z} h_{s} h_{z} \dot{E}_{t s} \\
& -\frac{3}{35} \varepsilon h_{q} h_{l} \frac{\hat{E}_{z s}}{\Delta} Y_{q l z s j k} \dot{E}_{i k} \\
& \left.-\frac{3 a}{35} \varepsilon h_{w} h_{l} \frac{\hat{E}_{m p}}{\Delta} Y_{w l m p j k} \varepsilon_{i q k} \varepsilon_{q t z} h_{s} h_{z} \dot{E}_{t s}\right]
\end{aligned}
$$

or

$$
\begin{aligned}
\dot{\sigma}_{i j}= & \frac{n k D^{3}}{2} \frac{\mu}{2-v}\left(\frac{\Delta}{3}\right)^{1 / 2} \\
& \times\left[\left(\frac{5-2 \varepsilon}{15}+\frac{14-20 \varepsilon}{105} \frac{\gamma}{\Delta}\right)\left(\delta_{i s} \delta_{k j}+\delta_{s j} \delta_{k i}\right)\right. \\
& +\left(\frac{5-2 \varepsilon}{15}+\frac{14-20 \varepsilon}{105} \frac{\gamma}{\Delta}\right) a \\
& \times\left(\delta_{i s} h_{j} h_{k}+\delta_{s j} h_{i} h_{k}+\delta_{j k} h_{i} h_{s}+\delta_{i k} h_{s} h_{j}\right) \\
& \left.+\left(\frac{4 \varepsilon}{5}-\frac{(1-\varepsilon) 4 \gamma}{5 \Delta} a-\frac{44 \varepsilon \gamma}{35 \Delta} a\right) h_{j} h_{k} h_{s} h_{i}\right] \dot{E}_{k s} .
\end{aligned}
$$

In the case of an isotropic aggregate, $\hat{\mathbf{E}}=\mathbf{0}, \varepsilon=0$, and the elastic moduli are

$$
\bar{G}=\frac{n \mu D^{3}}{4-2 v} \frac{\hat{k}}{3}\left(\frac{\Delta_{0}}{3}\right)^{1 / 2},
$$

and

$$
\bar{\Theta}=\frac{n \mu D^{3}}{4-2 v} \frac{2 \hat{k}}{9}\left(\frac{\Delta_{0}}{3}\right)^{1 / 2} .
$$

\section{APPENDIX B}

Here, we provide more details about the ratio in Eqs. (16)(18). In Ref. [33], it is shown that the difference between the EMT prediction and the numerical simulation is a fluctuation that should be added to the average deformation to properly describe the kinematics of contacting particles. In particular, when a perturbation in shear is considered, fluctuations in spin, rather than fluctuations in particle translation, are crucial. On the other hand, when a perturbation in pressure is considered, for example, in the case of the bulk modulus, fluctuations in spin are negligible, and a small fluctuation in translation occurs. The nature of the response of the aggregate makes it reasonable to normalize the shear moduli of an anisotropic aggregate by the shear modulus of an equivalent isotropic aggregate. The same applies to the bulk modulus. That is, a proper normalization mitigates the influence of the fluctuations.
[1] H. M. Jaeger, S. R. Nagel, and R. P. Behringer, Rev. Mod. Phys. 68, 1259 (1996).

[2] P.-G. de Gennes, Rev. Mod. Phys. 71, S374 (1999).

[3] P.-E. Peyneau and J.-N. Roux, Phys. Rev. E 78, 041307 (2008).

[4] L. Geng, G. Reydellet, E. Clément, and R. P. Behringer, Physica D 182, 274 (2003).

[5] C. Goldenberg and I. Goldhirsch, Phys. Rev. Lett. 89, 084302 (2002).

[6] J. R. F. Arthur and B. K. Menzies, Geotechnique 22, 115 (1972).

[7] M. Oda, Soils Found. 12, 17 (1972).

[8] M. Oda, H. Kazama, and J. Konishi, Mech. Mater. 28, 103 (1993).

[9] S. Nemat-Nasser, J. Mech. Phys. Solids 48, 1541 (2002).

[10] S. Nemat-Nasser and J. Zhang, Int. J. Plast. 18, 531 (2002).
[11] X. S. Li and Y. F. Dafalias, J. Geotech. Geoenviron. Eng. 128, 868 (2002).

[12] B. Cambou, P. Dubujet, and C. Nouguier-Lehon, Mech. Mater. 36, 1185 (2004).

[13] S. Luding, J. Phys.: Condens. Matter 17, S2623 (2005).

[14] Z. X. Yang, X. S. Li, and J. Yang, Geotechnique 58, 237 (2008).

[15] M. Kuhn, Mech. Mater. 42, 827 (2010).

[16] C. S. Chang and Z.-Y. Yin, J. Eng. Mech. 136, 830 (2010).

[17] W. Wu, Int. J. Numer. Analyt. Meth. Geomech. 22, 921 (1998).

[18] Y. C. Chen, I. Ishibashi, and J. T. Jenkins, Geotechnique 38, 25 (1988).

[19] M. M. Mehrabadi and S. Nemat-Nasser, Mech. Mater. 2, 155 (1983). 
[20] Y. Khidas and X. Jia, Phys. Rev. E 81, 021303 (2010).

[21] T. S. Majmudar and R. P. Behringer, Nature (London) 435, 1079 (2005).

[22] T. S. Majmudar, M. Sperl, S. Luding, and R. P. Behringer, Phys. Rev. Lett. 98, 058001 (2007).

[23] R. Kuwano and R. J. Jardine, Geotechnique 52, 727 (2002).

[24] T. K. Agarwal and I. Ishibashi, in Advances in Micromechanics of Granular Materials, edited by H. H. Shen et al. (Elsevier, Amsterdam, 1992), pp. 51-60.

[25] A. V. Abelev and P. V. Lade, J. Eng. Mech. 129, 160 (2003).

[26] A. V. Abelev and P. V. Lade, J. Eng. Mech. 129, 167 (2003).

[27] A. Ezaoui and H. Di Benedetto, Geotechnique 59, 621 (2009).

[28] F. Calvetti, G. Combe, and J. Lanier, Mech. Cohesive-Frict. Mater. 2, 121 (1997).

[29] I. Ishibashi, T. K. Agarwal, and S. A. Ashraf, in Proceedings of the 1st US Conference on Discrete Element Methods (DEM), Golden, CO, 1989 (Colorado School of Mines, Golden, CO, 1989).

[30] P. J. Digby, J. Appl. Mech. 48, 803 (1981).

[31] K. Walton, J. Mech. Phys. Solids 35, 213 (1987).

[32] J. Jenkins, D. Johnson, L. La Ragione, and H. A. Makse, J. Mech. Phys. Solids 53, 197 (2005).

[33] L. La Ragione and J. T. Jenkins, Proc. R. Soc. London, Ser. A 463, 735 (2007).

[34] J. T. Jenkins, in Modern Theory of Anisotropic Elasticity and Its Applications, edited by J. Wu, T. C. T. Ting, and D. M. Barnett (Society for Industrial and Applied Mathematics, Philadelphia, 1991), pp. 368-377.
[35] C. S. Chang, S. J. Chao, and Y. Chang, Int. J. Solids Struct. 32, 1989 (1995).

[36] F. Emeriault and B. Cambou, Int. J. Solids Struct. 33, 2591 (1996).

[37] I. Ishibashi, T. K. Agarwal, and S. A. Ashraf, International Conference on 1 st Discrete Element Methods, Golden, CO, 1989 (Colorado School of Mines, Golden, CO, 1989).

[38] X. Li, H. S. Yu, and X. S. Li, Int. J. Solids Struct. 46, 4331 (2009).

[39] P. A. Cundall and O. D. L. Strack, Geotechnique 29, 47 (1979).

[40] H. A. Makse, N. Gland, D. L. Johnson, and L. Schwartz, Phys. Rev. Lett. 83, 5070 (1999); H. A. Makse, N. Gland, D. L. Johnson, and L. Schwartz, Phys. Rev. E 70, 061302 (2004).

[41] S. Torquato, Random Heterogeneous Materials, 1st ed. (Springer-Verlag, New York, 2001).

[42] V. Magnanimo, L. La Ragione, J. T. Jenkins, P. Wang and H. A. Makse, Europhys. Lett. 81, 34006 (2008).

[43] A. E. H. Love, A Treatise on the Mathematical Theory of Elasticity (Cambridge University Press, Cambridge, UK, 1942).

[44] J. T. Jenkins, in Micromechanics of Granular Materials, edited by M. Satake and J. T. Jenkins (Elsevier, Amsterdam, 1988), pp. 245-252.

[45] K. Kanatani, Int. J. Eng. Sci. 22, 149 (1984).

[46] S. C. Cowin, Mech. Mater. 4, 137 (1985).

[47] I. Agnolin and J. N. Roux, Phys. Rev. E 76, 061304 (2007).

[48] T. K. Agarwal, Ph.D. thesis, Old Dominion University, 1991.

[49] J. T. Jenkins, P. A. Cundall, and I. Ishibashi, in Powder and Grains, edited by Biarez and Gourvès (Balkema, Rotterdam, 1989), pp. 257-264. 